\title{
Advances in Regenerative Medicine: From Stem Cells to Organoids
}

\author{
Jeanne Adiwinata Pawitan1,2,3 \\ ${ }^{1}$ Department of Histology, Faculty of Medicine Universitas Indonesia, Jakarta, Indonesia \\ ${ }^{2}$ Stem Cell Medical Technology Integrated Service Unit, Dr. Cipto Mangunkusumo General Hospital/Faculty of Medicine \\ Universitas Indonesia, Jakarta, Indonesia \\ ${ }^{3}$ Stem Cell and Tissue Engineering Research Center, Indonesia Medical Education and Research Institute (IMERI), Faculty of \\ Medicine Universitas Indonesia, Jakarta, Indonesia \\ Email: jeanneadiwip@gmail.com
}

How to cite this paper: Pawitan, J.A. (2018) Advances in Regenerative Medicine: From Stem Cells to Organoids. Journal of Biosciences and Medicines, 6, 128-136. https://doi.org/10.4236/jbm.2018.612012

Received: December 15, 2018

Accepted: December 28, 2018

Published: December 31, 2018

\begin{abstract}
Stem cells have moved from lab to bedside, and many initial studies showed promising results. Therefore big companies are entering the business. However, most initial studies did not used controls to make sure of the efficacy of stem cells. Many phase-1 studies showed safety of stem cell therapies, when precaution measures were adapted. However, efficacy needs to be proven by randomized controlled trials (RCT) to exclude placebo effects. Recently, various RCTs for various conditions have been done with various contradictory results. Therefore, a meta-analysis is very useful to know whether a stem cell therapy really work for a certain condition. As various centres used various type of stem cells, various dose, and route of application, as well as different outcome measures with various results for one certain condition, sometimes it is difficult to conduct a meta-analysis when there is high heterogeneity, which is like pooling "apples" with "oranges" and "avocado" that will lead to a misleading conclusion. In many cases, where the studies are highly heterogeneous, and the heterogeneity can't be identified, then a descriptive systematic review is the best solution to take a conclusion which protocol is the best and valuable to be standardized. Formerly it was believed that stem cells that are given to patients work by differentiating into the needed cells, and thus replacing damaged cell. However, recent evidence showed that only a few stem cells homed to the desired area, while a large amount went to various areas that were remote from the damaged area. Even though they were trapped in remote areas, the stem cells still exerted beneficial effects by remote signalling and secretion of various beneficial factors. Therefore, there are attempts to produce stem cell secretomes/metabolites to replace the stem cells, as metabolites are easier to handle and transported compared to the
\end{abstract}


cells themselves. In addition, various studies worked on substitute tissue/organs "ex vivo" to be transplanted to replace a damaged organ. There are various means to produce a tissue/an organ/organoid "ex vivo" (tissue engineering) by using various stem cells, scaffold, and soluble factors, in various vessels from static vessel to bioreactors, and "on chips". Though these attempts are in the initial stage, but some translational animal studies have been done. A more usual use of these "ex vivo" developed tissues/organs/organoids is for drug testing, such as toxicity testing, and for studying the mechanism of certain diseases that is directed toward the development of a cure of the diseases. In conclusion, many stem cell therapies have entered RCTs, but no standardized and approved protocol has been established, while organoids are usually used for drug testing and studying the mechanism of certain diseases.

\section{Keywords}

Regenerative Medicine, Stem Cell, RCT, Secretomes, Metabolites, Organoid, Scaffold

\section{Introduction}

Stem cells are very promising in regenerative medicine; thus stem cell therapy has moved from lab to bedside, and many initial studies showed promising results. Therefore big companies are entering the business [1]. Various kinds of stem cells especially mesenchymal stem cells (MSCs) from various sources have entered mass production. Mesenchymal stem cells are very promising to be developed as they showed immunomodulation properties [2], so that they are more suitable in allogeneic use compared to hematopoietic stem cells. However, production process, consumables that are used and culture procedures and conditions are different between companies, thus not standardized [3]. These mass products might be different from the stem cells that were produced and used in hospital based initial clinical trials, which did not over expand the stem cells. Many phase-1 studies showed safety of stem cell therapies, when precaution measures were adapted. However, to prove that the efficacy was due to the given stem cells, randomized controlled trials (RCT) were needed to exclude placebo effects. Recently, various RCTs that used various kinds of stem cells for various conditions have been done with various contradictory results [4] [5]. Therefore, meta-analyses are needed to conclude which protocol is the best and valuable to follow and to be standardized for a certain disease.

Formerly it was believed that stem cells that are given to patients work by differentiating into the needed cells, and thus replacing damaged cell. However, recent evidences showed that stem cells work through paracrine secretions to induce healing [6]. Therefore, recently many studies work on stem cell secretions that are called secretomes/metabolites [7]. Various animal studies showed that 
secretomes exerted beneficial result for certain diseases/conditions, which caused big pharmacies to enter in this area [8].

In addition, various studies worked on substitute tissue/organs/organoids "ex vivo" to be transplanted to replace a damaged organ. Some of the engineered tissue/organs/organoids were transplanted in animals and there are hopes that in the future, these engineered organoids might mimic the real organ and could answer the problems of organ/donor shortage [9] [10] [11]. Therefore this review addressed our simple methods for mesenchymal stem cell isolation and expansion using xeno-free materials so that they are safe to be used in clinical trials, the importance of meta-analysis on stem cell use in various conditions/diseases to get insights on the best protocol, stem cell metabolite production, and organoid studies.

\section{Simple Methods for MSC Isolation and Expansion}

Mesenchymal stem cells can be isolated from various tissues. Initially most centres used bone marrow (BM) derived MSCs, but later, the use of adipose tissue (AT) derived MSCs is increasing due to the easier and less invasive collection procedure. For allogeneic purposes, umbilical cord (UC) MSCs are preferred, as they do not pose any risk to the donors, and they have more plasticity compared to BM or AT derived MSCs. We have developed simple and effective methods in isolation and culture of BM and UC MSCs, and a simple washing method for liposuction derived AT (lipoaspirate), to be processed further to yield MSCs [12] [13] [14].

\subsection{Simple Method to Isolate BM MSCs}

A previous popular method to isolate BM MSC was by using a separation medium such as Ficoll and centrifugation, followed by washing steps to get the mononuclear cells (MNCs), and finally culturing the MNCs. We have developed a simpler method that do not need a separation medium, where the BM is simply washed in complete medium that contains $10 \%$ in house processed platelet lysate, and upper part of the pellet, which contained all the cells including erythrocytes, is cultured in a flask. It is impossible to observe cells attachment in the flask culture due to the abundance of erythrocytes. Therefore, to know when to wash the flask culture and the first medium change, the supernatant that contains few cells is centrifuged and the pellet is re-suspended in $1 \mathrm{~mL}$ complete medium and cultured in a well of a 12-well plate. When the cells in the well begin to attach, it is time to wash and change the medium of flask culture. Further medium change is done every two-three days until one of the clone is $90 \%$ confluent, when the cells can be harvested. Our method is more economical as we do not use Ficoll, and saves time as some steps of centrifugation can be reduced [12].

\subsection{Simple Method to Isolate UC MSCs}

Previous popular method of UC MSC isolation is by enzymatic method using 
two steps of digestion by collagenase and hyaluronidase. We have developed a multiple harvest explant method, which do not use enzymes, and thus more economical. By this method, the UC is washed, the umbilical artery and vein is discarded, and the UC is minced in small pieces of $2-5 \mathrm{~mm}$, and the pieces are put in wells of 24-well plate, one piece per well. The pieces are immersed in minimal amounts of complete medium to prevent them from floating. The culture is observed daily, and medium is added when necessary to prevent drying. When cells begin to sprout, medium can be added to be $0.5 \mathrm{~mL} /$ well. When the cells are $90 \%$ confluent, they are harvested. After harvest the explant is cultured again to yield more cells [13].

\subsection{Simple Washing Method of Lipoaspirate}

Previous washing of lipoaspirate was done by multi step washing and centrifugation; therefore it was time consuming and needed lots of washing medium. Lipoaspirate needs to be washed thoroughly to remove anaesthetics and tumescent solution, which are toxic to cells, free lipids that are released by damaged adipocytes, and erythrocytes. We have introduced a simpler method of washing by using a stainless coffee filter. In brief, the lipoaspirate is poured into the filter that retains it. Then the filter is soaked into a washing buffer containing bowl and stirred using a small stainless tea spoon, so that unwanted materials are going out via the filter pores, while the lipoaspirate is retained. This step is done several times until the buffer is clear. The clean lipoaspirate is then ready for further processing [14].

\subsection{Characterization of MSCs}

Our BM, AT and UC derived MSCs were characterized for their surface markers and differentiation capacity. All of our MSCs met the ISCT criteria in term of their surface markers, except AT derived MSCs that contained a substantial number of CD34, which was reduced by passaging [15]. Moreover, all of our MSCs showed differentiation capacity into three lineages namely into chondrogenic, osteogenic and adipogenic lineages, though the lipid droplets of UC derived MSCs were much smaller than those of BM and AT derived MSCs [16].

\section{Importance of Meta-Analysis on Stem Cell Use}

Our MSCs have been used in various phase-1 clinical trials that are on-going. Until to date, no MSC related adverse events have been reported. Globally, various phase- 1 studies showed safety of stem cell therapies, when precaution measures were adapted. However, a compilation report on published studies and various mass media report on adverse events in unproven stem cell intervention showed 35 reports of stem cell related adverse events [17].

Some of the phase-1 clinical trials using our MSCs showed promising results and were reported as case reports [18]. However, efficacy needs to be proven by randomized controlled trials (RCT) to exclude placebo effects, or the possibility 
of natural healing process of the disease. Recently, various RCTs for various conditions have been done with various contradictory results. Most hospital based efficacy studies using controls showed beneficial results of MSCs for graft versus host disease, but a large multi-centre study showed no difference between treatment and controls [19].

Therefore, a meta-analysis is very useful to know whether a stem cell therapy really work for a certain condition. Various centres used various types of stem cells from various sources, various dose and vehicles, and route of application, as well as various numbers of repeats. Moreover, there are different outcome measures with various results for one certain condition, which make it difficult to conduct a meta-analysis when there is high heterogeneity, which is like pooling "apples" with "oranges" and "avocado" that will lead to a misleading conclusion. Therefore, when the studies are highly heterogeneous, and the heterogeneity can't be identified, then a descriptive systematic review is the best solution to take a conclusion which protocol is the best [20]. The protocol that gives the best result is valuable to be standardized.

\section{Stem Cell Metabolite Production}

Formerly it was believed that stem cells that are given to patients work by homing to the damaged area, then differentiating into the needed cells, and thus replacing damaged cell to cause the cure of the disease. However, recent evidence showed that only a few stem cells homed to the desired area, while a large amount went to various areas that were remote from the damaged area, mostly in the lungs, spleen, and bone marrow. Even though they were trapped in remote areas, the stem cells still exerted beneficial effects by remote signalling and secretion of various beneficial factors, such as growth factors, beneficial cytokines and chemokines, microvesicles and exosomes (Figure 1) [6].

Therefore, there are attempts to produce stem cell secretomes/metabolites to replace the stem cells themselves [7]. There are various methods to produce stem

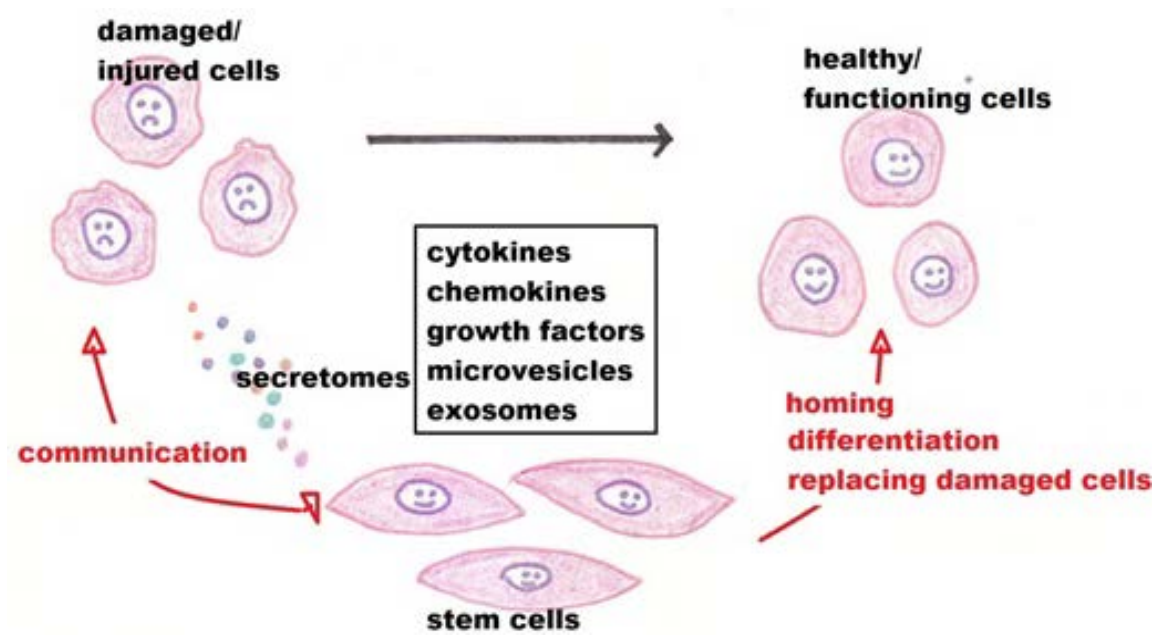

Figure 1. Mechanism of action of stem cells in repairing damaged tissues/organs. 
cell metabolites in terms of the type of cells, cell source, culture medium, culture condition, culture duration, and processing of the metabolites. Various studies used various types of stem cells to produce stem cells metabolites such as embryonic stem cells, induced pluripotent stem cells, MSCs from various sources such as BM, AT, UC, UC blood, etc. Moreover, some studies challenged the metabolite producing cells by co-culturing with injured cells to be repaired before producing the metabolites. Some studies used serum free basal medium, while others used serum, growth factor or platelet lysate containing basal medium; the basal medium were also variable. Culture condition to produce stem cell metabolites can be normoxia $\left(20 \% \mathrm{O}_{2}\right)$, or hypoxia $\left(1 \%-5 \% \mathrm{O}_{2}\right)$, and culture duration might range from 1 - 4 days [21].

Processing of metabolites varies between studies. Pioneering studies used whole metabolites that were only filtered to remove remaining cells and debris; some other studies concentrated the metabolites with various power of concentration, while more recent studies separated the various contents in metabolites, such as microvesicles and exosomes [22]. We used whole metabolites in animal studies and showed beneficial effects in third degree burn wound [23] [24].

In the future, as metabolites are easier to handle and transported compared to the cells themselves, which need cryopreservation and culture facilities to re-culture thawed stem cells, metabolites are easier and more readily to be developed into drugs [7].

\section{Organoid Studies}

Recently, various studies used stem cells to develop substitute tissue/organs "ex vivo" with various degree of success, which the ultimate goal is to be transplanted to replace a damaged organ [10]. There are various means to produce a tissue/an organ/organoid "ex vivo" (tissue engineering) by combining various stem cells, scaffold, and soluble factors. Many studies used embryonic stem cells or induced pluripotent stem cells, but there are also studies that used adult stem cells, such as MSCs from various sources. For scaffolds, there are various choices of scaffolds. Natural scaffolds are more biocompatible and friendly to the seeded cells, but for adjusting the biomechanics and shape of the tissue/organ to be developed, synthetic scaffold might have more advantages compared to natural scaffold. Therefore, careful and smart combination of synthetic and natural materials will determine the success of engineered tissue/organ development [9]. From all scaffolds, theoretically natural organ derived scaffold is the best. Natural organ derived scaffolds can be obtained by decellularization of an organ using various enzymes and chemicals [25].

Besides cells and scaffolds, soluble factors such as various growth factors and cytokines are also needed to stimulate the orchestra of developmental processes. Therefore knowledge of developmental biology is very important to give guidance in the sequence and timing of growth factor and cytokine administration [9]. 
In some studies, tissue engineering was done by combining cells that formed clusters mimicking an organ that is called an organoid [11]. Our on-going study is to develop liver organoids by combining various types of cells (hepatocytes, MSCs, CD34 from umbilical cord blood, and hepatic stellate cells) in different ratio and media.

Tissue/organs/organoids are usually developed in various vessels from static vessel to bioreactors, and "on chips" [25] [26] [27]. Bioreactors with fluidic system or chips with microfluidic system can mimic the circulation, thus theoretically can develop the organoids better than using static vessels.

Though attempts to develop tissues/organs/organoids are in the initial stage, some translational animal studies have been done [28]. However, a more usual use of these "ex vivo" developed tissues/organs/organoids is for drug testing, such as toxicity testing, and for studying the mechanism of certain diseases that is directed toward the development of a cure of the diseases [27].

In conclusion, many stem cell therapies have entered RCTs, but no standardized and approved protocol has been established, while organoids are usually used for drug testing and studying the mechanism of certain diseases.

\section{Acknowledgements}

This work was supported by a research grant from the Ministry of Research, Technology and Higher Education of the Republic of Indonesia, Hibah PUSN 2018, contract no.554/UN2.R3.1/HKP05.00/2018.

\section{Conflicts of Interest}

The author declares that there are no conflicts of interest.

\section{References}

[1] Mesoblast (2018) Corporate Backgrounder. http://investorsmedia.mesoblast.com/phoenix.zhtml?c=187006\&p=irol-homeProfile \& $\mathrm{t}=$ \&id $=\&$

[2] Gao, F., Chiu, S.M., Motan, D.A., Zhang, Z., Chen, L., Ji, H.L., Tse, H.F., Fu, Q.L. and Lian, Q. (2016) Mesenchymal Stem Cells and Immunomodulation: Current Status and Future Prospects. Cell Death Dis, 7, e2062.

https://doi.org/10.1038/cddis.2015.327

[3] Smith, J.R., Pfeifer, K., Petry, F., Powell, N., Delzeit, J. and Weiss, M.L. (2016) Standardizing Umbilical Cord Mesenchymal Stromal Cells for Translation to Clinical Use: Selection of GMP-Compliant Medium and a Simplified Isolation Method. Stem Cells Int, 2016, 6810980. https://doi.org/10.1155/2016/6810980

[4] Lu, Y., Wang, Y., Lin, M., Zhou, J., Wang, Z., Jiang, M., and He, B. (2016) A Systematic Review of Randomised Controlled Trials Examining the Therapeutic Effects of Adult Bone Marrow-Derived Stem Cells for Non-Ischaemic Dilated Cardiomyopathy. Stem Cell Res Ther, 7, 186. https://doi.org/10.1186/s13287-016-0441-x

[5] Pawitan, J.A., Yang, Z., Wu, Y.N. and Lee, E.H. (2018) Towards Standardized Stem Cell Therapy in Type 2 Diabetes Mellitus: A Systematic Review. Current Stem Cell Research and Therapy, 13, 476-488. 
https://doi.org/10.2174/1574888X13666180502143657

[6] Hodgkinson, C.P., Bareja, A., Gomez, J.A. and Dzau, V.J. (2016) Emerging Concepts in Paracrine Mechanisms in Regenerative Cardiovascular Medicine and Biology. Circ Res, 118, 95-107. https://doi.org/10.1161/CIRCRESAHA.115.305373

[7] Vizoso, F.J., Eiro, N., Cid, S., Schneider, J. and Perez-Fernandez, R. (2017) Mesenchymal Stem Cell Secretome: Toward Cell-Free Therapeutic Strategies in Regenerative Medicine. Int JMol Sci, 18, E1852. https://doi.org/10.3390/ijms18091852

[8] Hildred, C. (2018) Explosion of Interest in Exosome Technologies and Companies Leading the Charge. https://www.bioinformant.com/exosome-technologies/

[9] Pawitan, J.A. (2011) Future Research in Adipose Stem Cell Engineering. In: Illouz, Y.-G. and Sterodimas, A., Eds., Adipose Stem Cells and Regenerative Medicine, Springer, Heidelberg, 257-272. https://doi.org/10.1007/978-3-642-20012-0_23

[10] Koh, C.J. and Atala, A. (2004) Tissue Engineering, Stem Cells, and Cloning: Opportunities for Regenerative Medicine. J Am SocNephrol, 15, 1113-1125. https://doi.org/10.1097/01.ASN.0000119683.59068.F0

[11] Huch, M., Knoblich, J.A., Lutolf, M.P. and Martinez-Arias, A. (2017) The Hope and the Hype of Organoid Research. Development, 144, 938-941. https://doi.org/10.1242/dev.150201

[12] Pawitan, J.A., Feroniasanti, L., Kispa, T., Dilogo, I.H., Fasha, I., Kurniawati, T. and Liem, I.K. (2014-2015) Simple Method to Isolate Mesenchymal Stem Cells from Bone Marrow Using Xeno-Free Material: A Preliminary Study. Int J Pharm Tech Res, 7, 354-359.

[13] Pawitan, J.A., Liem, I.K., Budiyanti, E., Fasha, I., Feroniasanti, L., Jamaan, T. and Sumapradja, K. (2014) Umbilical Cord Derived Stem Cell Culture: Multiple-Harvest Explant Method. Int J PharmTech Res, 6, 1202-1208.

[14] Pawitan, J.A., Liem, I.K., Suryani, D., Bustami, A. and Purwoko, R.Y. (2013) Simple Lipoaspirate Washing Using a Coffee Filter. Asian Biomedicine, 7, 333-338.

[15] Pawitan, J.A., Wulandari, D., Suryani, D., Damayanti, L., Purwoko, R.Y. and Liem, I.K. (2013) Flow Cytometry Analysis of Adipose Tissue Derived Stem Cells That Were Cultured in Various Media. Int J Pharm Tech Res, 5, 1301-1306.

[16] Pawitan, J.A., Kispa, T., Mediana, D., Goei, N., Fasha, I., Liem, I.K. and Wulandari, D. (2015) Simple Production Method of Umbilical Cord Derived Mesenchymal Stem Cell Using Xeno-Free Materials for Translational Research. J Chem Pharm Res, 7, 652-656.

[17] Bauer, G., Elsallab, M. and Abou-El-Enein, M. (2018) Concise Review: A Comprehensive Analysis of Reported Adverse Events in Patients Receiving Unproven Stem Cell-Based Interventions. Stem Cells Transl Med, 7, 676-685. https://doi.org/10.1002/sctm.17-0282

[18] Dilogo, I.H., Primaputra, M.R.A., Pawitan, J.A. and Liem, I.K. (2017) Modified Masquelet Technique using Allogeneic Umbilical Cord-Derived Mesenchymal Stem Cells For Infected Non-Union Femoral Shaft Fracture with a $12 \mathrm{~cm}$ Bone Defect: A Case Report. Int J Surg Case Report, 34, 11-16. https://doi.org/10.1016/j.ijscr.2017.03.002

[19] Galipeau, J. ()2013 The Mesenchymal Stromal Cells Dilemma-Does a Negative Phase III Trial of Random Donor Mesenchymal Stromal Cells in Steroid-Resistant Graft-Versus-Host Disease Represent A Death Knell or a Bump in the Road? Cytotherapy, 15, 2-8. https://doi.org/10.1016/j.jcyt.2012.10.002

[20] Wong, G., Sharma, A., Teixeira-Pinto, A. and Howell, M. (2018) Systematic Reviews 
and Meta-Analyses in Transplantation: Challenges and Pitfalls? Transplantation, 102, 1415-1418. https://doi.org/10.1097/TP.0000000000002314

[21] Pawitan, J.A. (2014) Prospect of Stem Cell Conditioned Medium in Regenerative Medicine. Biomed Research International, 2014, 965849. https://doi.org/10.1155/2014/965849

[22] Pachler, K., Lener, T., Streif, D., Dunai, Z.A., Desgeorges, A., Feichtner, M., Öller, M., Schallmoser, K., Rohde, E. and Gimona, M. (2017) A Good Manufacturing Practice-Grade Standard Protocol for Exclusively Human Mesenchymal Stromal Cell-Derived Extracellular Vesicles. Cytotherapy, 19, 458-472. https://doi.org/10.1016/j.jcyt.2017.01.001

[23] Eryani, A., Sukmawati, D., Damayanti, L., Angmalisang, E.C. and Pawitan, J.A. (2018) The Healing Effect of Adipose-Derived Stem Cell Conditioned Medium on Burn Wound Model. Trends in Biomaterials and Artificial Organs,32, 18-25.

[24] Angmalisang, E.C., Sukmawati, D., Damayanti, L., Astheria, E. and Pawitan, J.A. (2018) Adipose-Derived Stem Cell Conditioned Medium Enhances Expression of Ephrin-B2 Related to Neovascularization in Burn Wound Tissue. Adv Sci Lett, 24, 6168-6172. https://doi.org/10.1166/asl.2018.12668

[25] Mazza, G., Rombouts, K., Rennie Hall, A., Urbani, L., VinhLuong, T., Al-Akkad, W., Longato, L., Brown, D., Maghsoudlou, P., Dhillon, A.P., Fuller, B., Davidson, B., Moore, K., Dhar, D., De Coppi, P., Malago, M. and Pinzani, M.. (2015) Decellularized Human Liver as a Natural 3D-Scaffold for Liver Bioengineering and Transplantation. Sci Rep, 5, 13079. https://doi.org/10.1038/srep13079

[26] Zhao, J., Griffin, M., Cai, J., Li, S., Bulter, P.E.M. and Kalaskar, .DM. (2016) Bioreactors for Tissue Engineering: An Update. Biochemical Engineering Journal, 109, 268-281. https://doi.org/10.1016/j.bej.2016.01.018

[27] Takebe, T., Zhang, B. and Radisic, M. (2017) Synergistic Engineering: Organoids Meet Organs-on-a-Chip. Cell Stem Cell, 21, 297-300. https://doi.org/10.1016/j.stem.2017.08.016

[28] Stevens, K.R., Scull, M.A., Ramanan, V., Fortin, C.L., Chaturvedi, R.R., Knouse, K.A., Xiao, J.W., Fung, C., Mirabella, T., Chen, A.X., McCue, M.G., Yang, M.T., Fleming, H.E., Chung, K., de Jong, Y.P., Chen, C.S., Rice, C.M. and Bhatia, S.N. (2017) In Situ Expansion of Engineered Human Liver Tissue in a Mouse Model of Chronic Liver Disease. Sci Transl Med, 9, eaah5505.

https://doi.org/10.1126/scitranslmed.aah5505 